\title{
Improved Color Satellite Image Segmentation Using Tsallis Entropy and Granular Computing
}

\author{
Jagan Kumar. N, Agilandeeswari. L, Prabukumar. M
}

School of Information Technology and Engineering, VIT University, Vellore, India

\begin{tabular}{l}
\hline \hline Article Info \\
\hline Article history: \\
Received Sep 17, 2017 \\
Revised Nov 12, 2017 \\
Accepted Nov 21, 2017 \\
\hline Keyword: \\
Cuckoo search \\
Granular computing \\
Optimize \\
Segmentation \\
Tsallis \\
\hline
\end{tabular}

\begin{abstract}
The research work is to improve the segmentation of the color satellite images. In this proposed method the color satellite image can be segmented by using Tsallis entropy and granular computing methods with the help of cuckoo search algorithm. The Tsallis and granular computing methods will used to find the maximum possibility of threshold limits and the cuckoo search will find the optimized threshold values based on threshold limit that is calculated by the Tsallis entropy and granular computing methods and the multilevel thresholding will used for the segmentation of color satellite images based on the optimized threshold value that will find by this work and these methods will help to select the optimized threshold values for multiple thresholding effectively.
\end{abstract}

Copyright () 2017 Institute of Advanced Engineering and Science. All rights reserved.

Corresponding Author:

Jagan kumar,

School of Information Technology and Engineering,

VIT University,

Vellore 632014, TN, India.

Email: kumarjagan02@gmail.com

\section{INTRODUCTION}

Color image segmentation is the essential process for an image. The splitting of image in to various parts is called image segmentation. The segmentation used to make the image to be understandable. The image segmentation is used to find the object and the background of an image by the help of boundaries like line, curve etc [1]. The thresholding is basic segmentation technique of images. Basically image object and background is differentiating by single value of threshold. The color image can be threshold based on their RGB values of an image. While distinguish an image into various different regions based on various threshold limits is called multilevel thresholding [6]. The image is differentiated by the single limit $\mathrm{T}$ and if gray value is greater than $\mathrm{T}$ is considered to be an object else other gray value are considered to be background. The basic technique is not given the good result when coming to images of remote sensing because of this there will be essential of multilevel thresholding [2].

The non-extensive entropy of image segmentation the thresholding technique used on various kinds of gray scale images. The optimization algorithm had given the better threshold value by using Bacterial foraging algorithm [1]. The Bacterial foraging algorithm was used Tsallis objective function for the multilevel thresholding and they had associated with GA and PSO [4] methods and it got better result and converges faster than the PSO and GA methods.

The unsupervised multilevel thresholding techniques for segmenting of cloud areas from weather satellite images [2]. In this approach generate several binary images from a set of predefined threshold values, then they extracted and mapped the contour of cloudy areas included the image sequence and they also used some of preprocessing approaches like Gaussian smoothing for noise reduction and enhanced contrast by histogram equalization $[9,10]$. The goal here is to determine a global and coarse threshold that separates the image to distinct classes, the background and the foreground. This paper focuses on a specific 
cloud segmentation method for image obtained by meteorological satellites [6,9] and this algorithm effectively extract the representative areas of clouds and solved the problems of over segmentation and under segmentation related to the global thresholding methods.

In the granular computing for the segmentation it has the following processes to achieve the thresholding [4]. The granules are characterized with RGB values and it should combine the 2 granules to get larger granule and finally, establish by the U operation on granules to get threshold t of granules.

The main objective of this paper is follows, the satellite images are captured by satellite (Remote sensing) from the space. The capturing the images by the use of the remote sensing devices (machines) that faces many problems in the resolution of the images. Those problems are follows

a. Many satellite images have been affected by the shadows of the cloud in the sky.

b. The whether condition and light have big changes over the satellite images.

c. Objects are blocked by trees and shadows surrounding object with similar colors i.e., roof tops.

These conditions can make the objects predictability impossible [1].

Based on the above details explanation the existing algorithms the image segmentation color images using multilevel thresholding approach that are a critical and challenging task and it takes huge number of line coding to done the segmentation and in multilevel thresholding has some problem when the level increases that will produce the good result and because of more levels in thresholding have also increased the computational cost higher and moreover the time of computational also increased.

In this proposed method improve the segmentation based on Tsallis entropy and granular computing methods with the help of CS optimization algorithm and the paper [6] have proved that the CS is the best optimization algorithm when compared to the others. The rest of the paper is organized as follows, section 2 will be explain about the concepts that are used in this algorithm, section 3 will be the proposed work, section 4 contains the experimental results and in section 5 will be the conclusion.

\section{RESEARCH METHOD}

In this section contains the explanation of the various methods that are used in the segmentation of the color satellite image.

\subsection{Tsallis Entropy}

The entropy is the measure the states logarithmically with significant probability of being occupied. The Tsallis entropy is also called non-extensive entropy. The advantage of the Tsallis entropy method is the use of global property and objective property of the images [2,5].

$$
N=\sum_{i=0}^{L-1} h(i) \quad \text { And } \quad P_{i}=\frac{h(i)}{N}
$$

a. $\quad$ L -gray level between $\{0,1,2, \ldots,(\mathrm{L}-1)\}$.

b. $h(i)$ pixels in gray level $i$ and it should in $0 \leq i \leq(\mathrm{L}-1)$.

c. $\mathrm{N}$ is total number of pixels in a given image.

d. $\mathrm{Pi}$ is probability valuation.

Thresholding for multilevel using Tsallis define blow.

$$
f(t)=\arg \max \left[S_{q}^{A}(t)+S_{q}^{B}(t)+\ldots . .+S_{q}^{M}(t)+(1-q) \cdot S_{q}^{A}(t) \cdot S_{q}^{B}(t) \cdot \ldots . . S_{q}^{M}(t)\right]
$$

Where,

$$
\begin{aligned}
& S_{q}^{A}(t)=\frac{1-\sum_{i=0}^{t_{1}-1}\left(\frac{P_{i}}{P^{A}}\right)^{q}}{q-1} ; \quad P^{A}=\sum_{i=0}^{t_{1}-1} P_{i} \quad ; \quad S_{q}^{B}(t)=\frac{1-\sum_{i=t_{1}}^{t_{2}-1}\left(\frac{P_{i}}{P^{B}}\right)^{q}}{q-1} ; \quad P^{B}=\sum_{i=t_{1}}^{t_{2}-1} P_{i} \quad \text { and } \\
& S_{q}^{M}(t)=\frac{1-\sum_{i=t_{M}}^{L-1}\left(\frac{P_{i}}{P^{M}}\right)^{q}}{q-1} ; \quad P^{M}=\sum_{i=t_{M}}^{L-1} P_{i}
\end{aligned}
$$

In the above formula, $\mathrm{t}_{1}, \mathrm{t}_{2}, \ldots \ldots, \mathrm{t}_{\mathrm{M}}$ are threshold levels and it should be in $\mathrm{t}_{1}<\mathrm{t}_{2}<\mathrm{t}_{3} \ldots \ldots .<\mathrm{t}_{\mathrm{M}}$ 


\subsection{Granular Computing (GrC)}

This algorithm is solving the various problems by using various methods and tools and this computing is processing over the granules. It takes the pixels of an image and combines the pixels to get the granules based on dimension that is given by user and the values contains in a granule are appear based on the probability of the pixels in the granule[1,12].

Roughentropy(l)=e/2[obj_roughness(l)loge(obj_roughness(l))+back_roughness(l) loge(back_roughness(l))];

Topt=Argmax $($ Roughentropy(1));

\subsection{Cuckoo search}

It is an optimization algorithm and it has been process same as cuckoo. The Cuckoo Search algorithm can find the new solution is attempted to be examine over the previously founded finest results $[2,5,6]$.

There are 3 steps as follows.

a. The cuckoo puts an egg in the nest that is randomly selected.

b. The finest nest contains the great quality of eggs.

c. The probability of finding destination nest by destination bird pa is belongs to $[0,1]$.

\section{PROPOSED ALGORITHM:}

In this algorithm the thresholding of multilevel is using for the color satellite image segment and for the optimization purpose the optimization algorithm called cuckoo search (CS) is used to discover the optimized thresholded values of the color satellite images that is supported by non_extensive entropy that is called Tsallis entropy and another supporting method called granular computing. The both the tsallis entropy and granular computing are combine and used to find the maximum possible levels of the thresholding for an image. Finally in experimental section, we have compared the quality between original image and result image by using following matrices MSE, PSNR, SSIM, and NCC.

The flow chart of the proposed algorithm is shown in Figure 1.

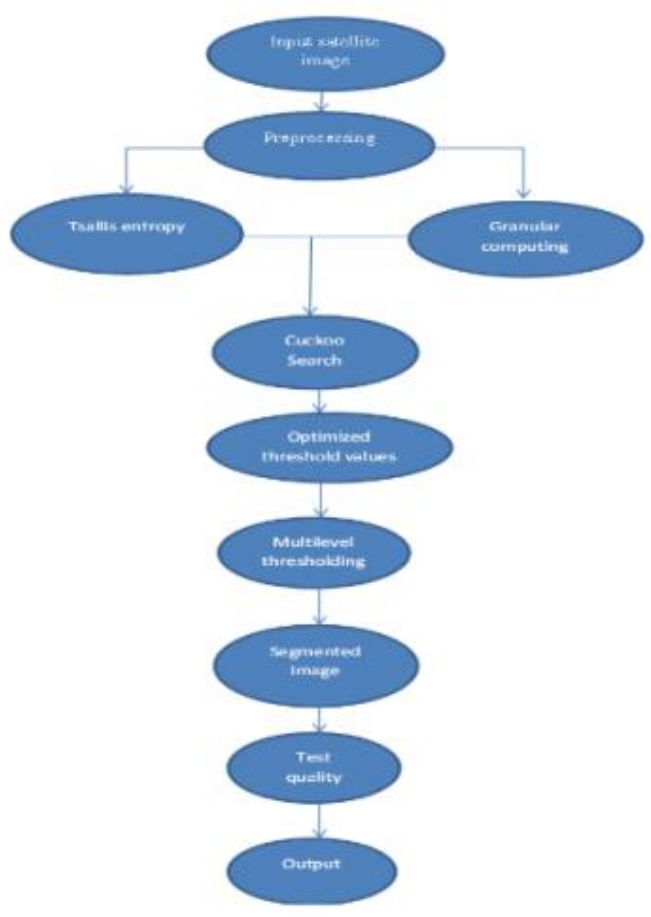

Figure 1. Flow Chart 
The above flow chart can be explained as follows. First select the input satellite image from the dataset and the input image format should be on .jpeg or .png format that is important. The preprocessing is used to slightly change the appearance of an image. Here in this work using the two preprocessing techniques. Increase or decrease the size of images using resize function in MATLAB and removing the noises of an image by the help of filter. To calculate optimize threshold value based 3 methods. The first two methods are used to find the maximum possibility of the values those are Tsallis entropy and granular computing based on formula $(2,4)$ and another one is the optimization algorithm that is called cuckoo search algorithm it is used to discovery the optimized threshold limit from the determined possibility.

\section{RESULTS AND ANALYSIS}

In this algorithm the satellite images or dataset are used from the NASA website (http://earthobservatory.nasa.gov/Images/?eocn=topnav\&eoci=images). The configuration requirement of this work is MATLAB 2013 software and the operating system should be windows 7 with hard disk capacity can be 30GB with basic keyboard. The following Table 1 contains optimized threshold values that are calculated by the proposed methodology.

Table 1. Optimized Threshold Values of Different Images with Different Threshold Levels

\begin{tabular}{|c|c|c|}
\hline Image No & Levels & Optimized Threshold Values \\
\hline \multirow{4}{*}{1} & 5 & $55,113,163,215,215$ \\
\hline & 6 & $51,95,95,165,165,230$ \\
\hline & 8 & $30,64,100,129,166,198,231$ \\
\hline & 12 & $33,65,81,109,138,152,173,194,217,243,243,243$ \\
\hline \multirow{4}{*}{2} & 5 & $49,103,103,166,214$ \\
\hline & 6 & $36,84,135,182,182,223$ \\
\hline & 8 & $24,57,88,130,159,196,228$ \\
\hline & 12 & $17,45,62,85,105,127,156,177,205,224,224,224$ \\
\hline \multirow{4}{*}{3} & 5 & $46,95,160,215,215$ \\
\hline & 6 & $26,90,90,182,182,223$ \\
\hline & 8 & $24,57,88,130,159,196,228$ \\
\hline & 12 & $17,45,62,85,105,127,156,177,205,224,224,224$ \\
\hline \multirow{4}{*}{4} & 5 & $41,41,90,153,209$ \\
\hline & 6 & $45,95,95,148,190,224$ \\
\hline & 8 & $23,51,104,140,174,200,200,232$ \\
\hline & 12 & $13,29,51,78,108,134,164,186,186,200,231,243$ \\
\hline \multirow{4}{*}{5} & 5 & $46,46,103,157,215$ \\
\hline & 6 & $36,78,123,165,165,219$ \\
\hline & 8 & $43,77,134,170,170,198,225$ \\
\hline & 12 & $30,46,71,90,113,151,170,185,211,236,236$ \\
\hline \multirow{4}{*}{6} & 5 & $52,108,166,212,212$ \\
\hline & 6 & $37,81,127,176,218,218$ \\
\hline & 8 & $70,70,70,107,136,164,191,228$ \\
\hline & 12 & $13,43,62,93,112,135,156,178,197,216,216,237$ \\
\hline \multirow{4}{*}{7} & 5 & $50,50,102,158,207$ \\
\hline & 6 & $44,87,87,126,171,218$ \\
\hline & 8 & $28,79,79,131,167,167,191,227$ \\
\hline & 12 & $33,33,60,60,74,90,112,139,166,186,221,239$ \\
\hline \multirow{4}{*}{8} & 5 & $41,57,110,165,215$ \\
\hline & 6 & $45,91,134,177,218,218$ \\
\hline & 8 & $52,52,52,89,129,155,192,233$ \\
\hline & 12 & $14,35,63,90,90,106,106,130,159,179,217,236$ \\
\hline
\end{tabular}




\subsection{The Original Images and its Histogram}

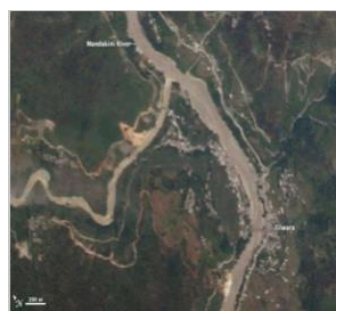

A1

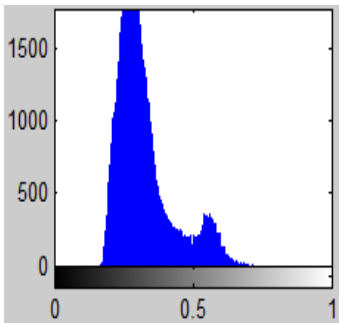

B1

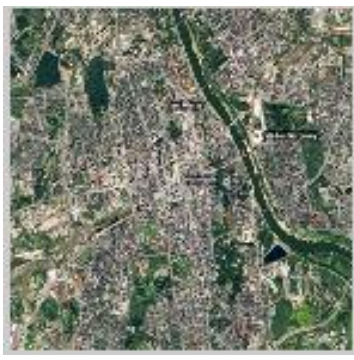

A5

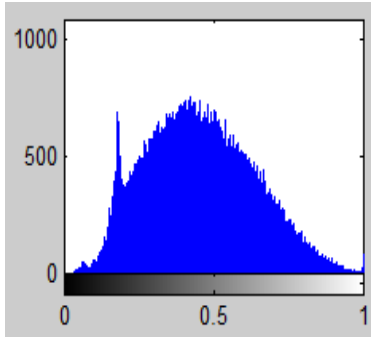

B5

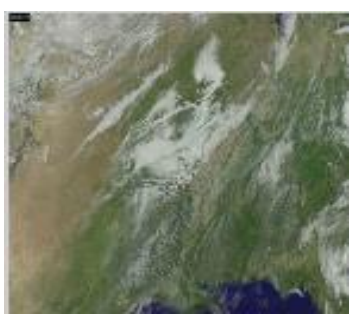

A2

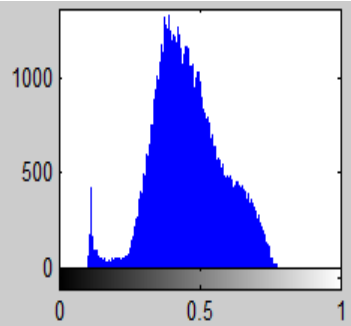

B2

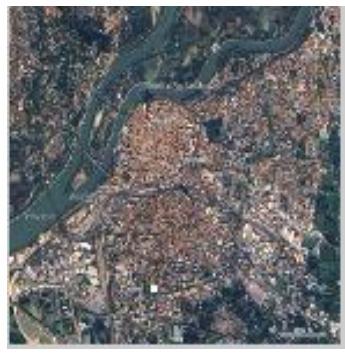

A6

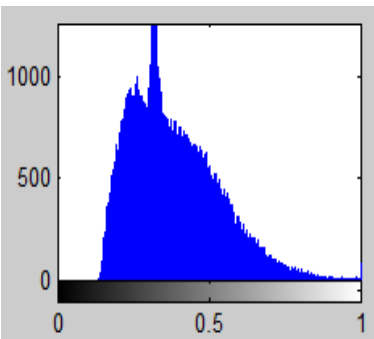

B6

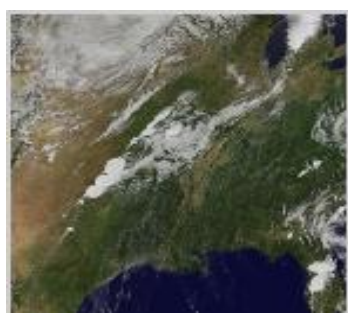

A3

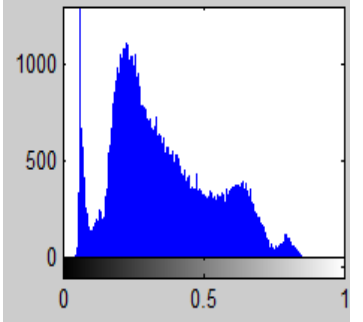

B3

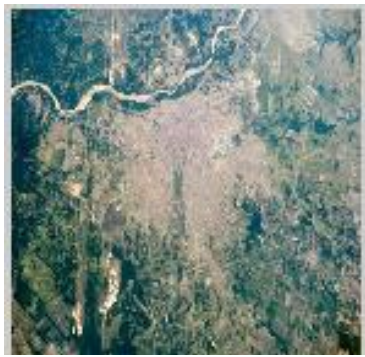

A7

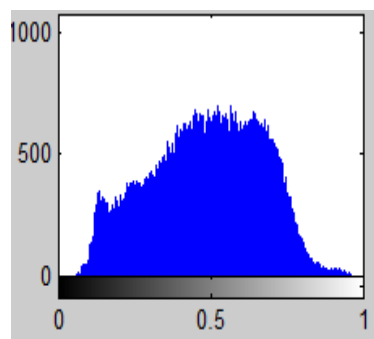

B7

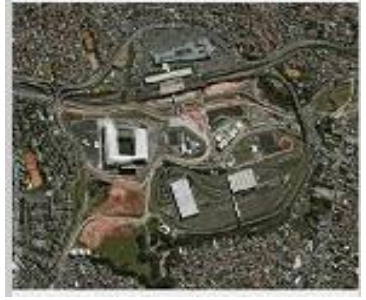

A4

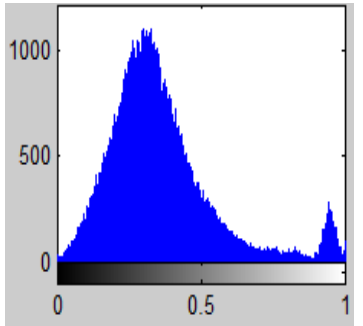

B4

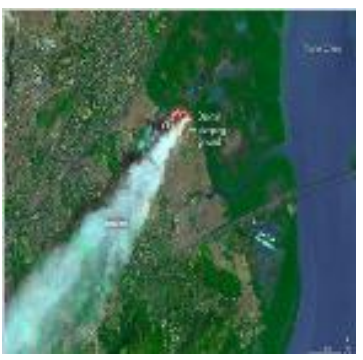

A8

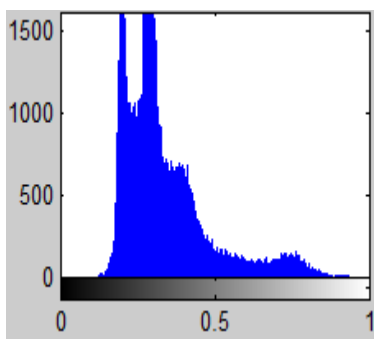

B8

Figure 2. In the Above Figures (A1-A8) are Original Satellite Images and Figures (B1-B8) are Their Equal Lent Histograms 


\subsection{Threshold Levels 5}
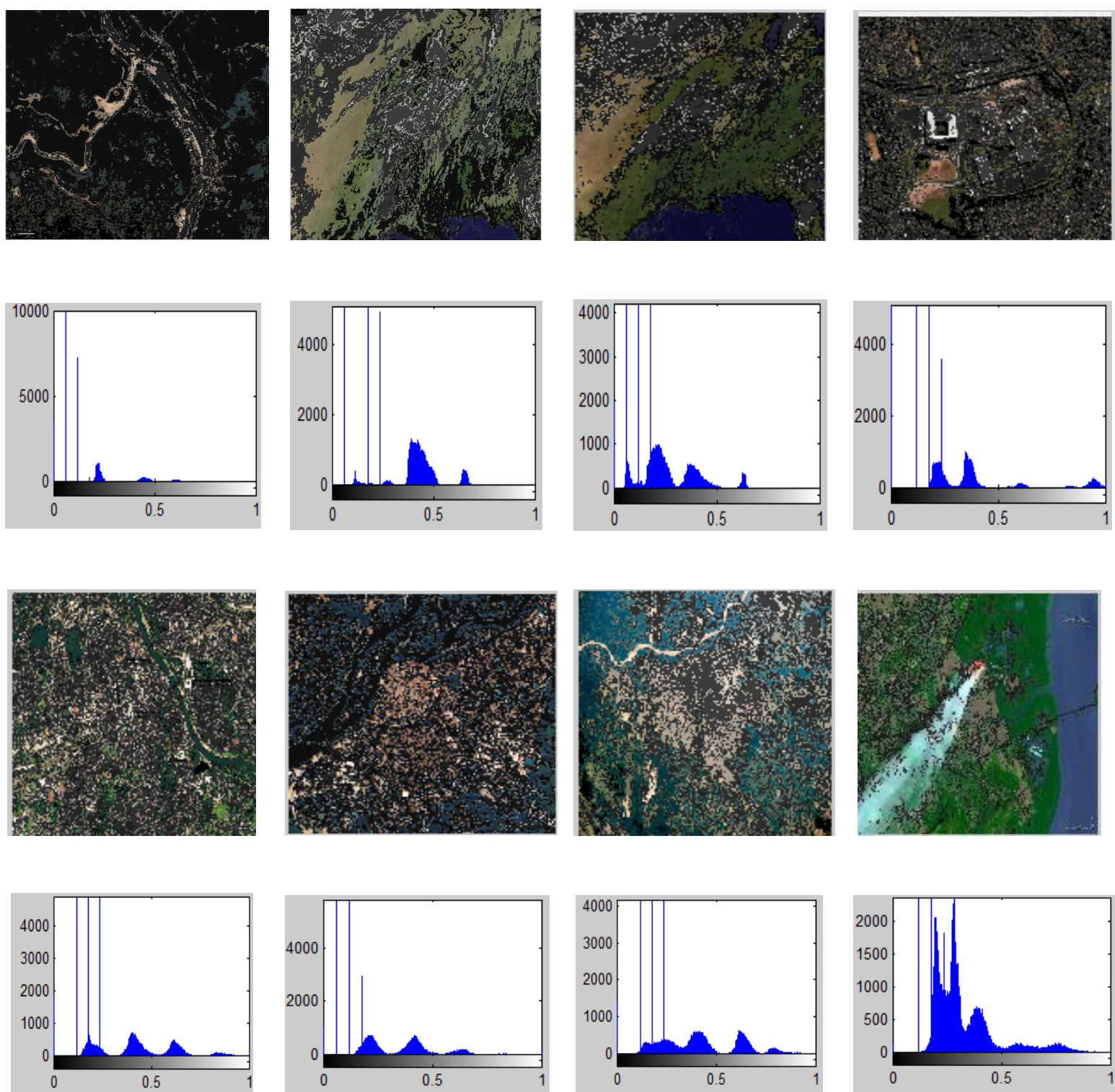

Figure 3. The 5 Levels Thresholding Based Segmented Images with Their Histograms 


\subsection{Threshold Levels 6}
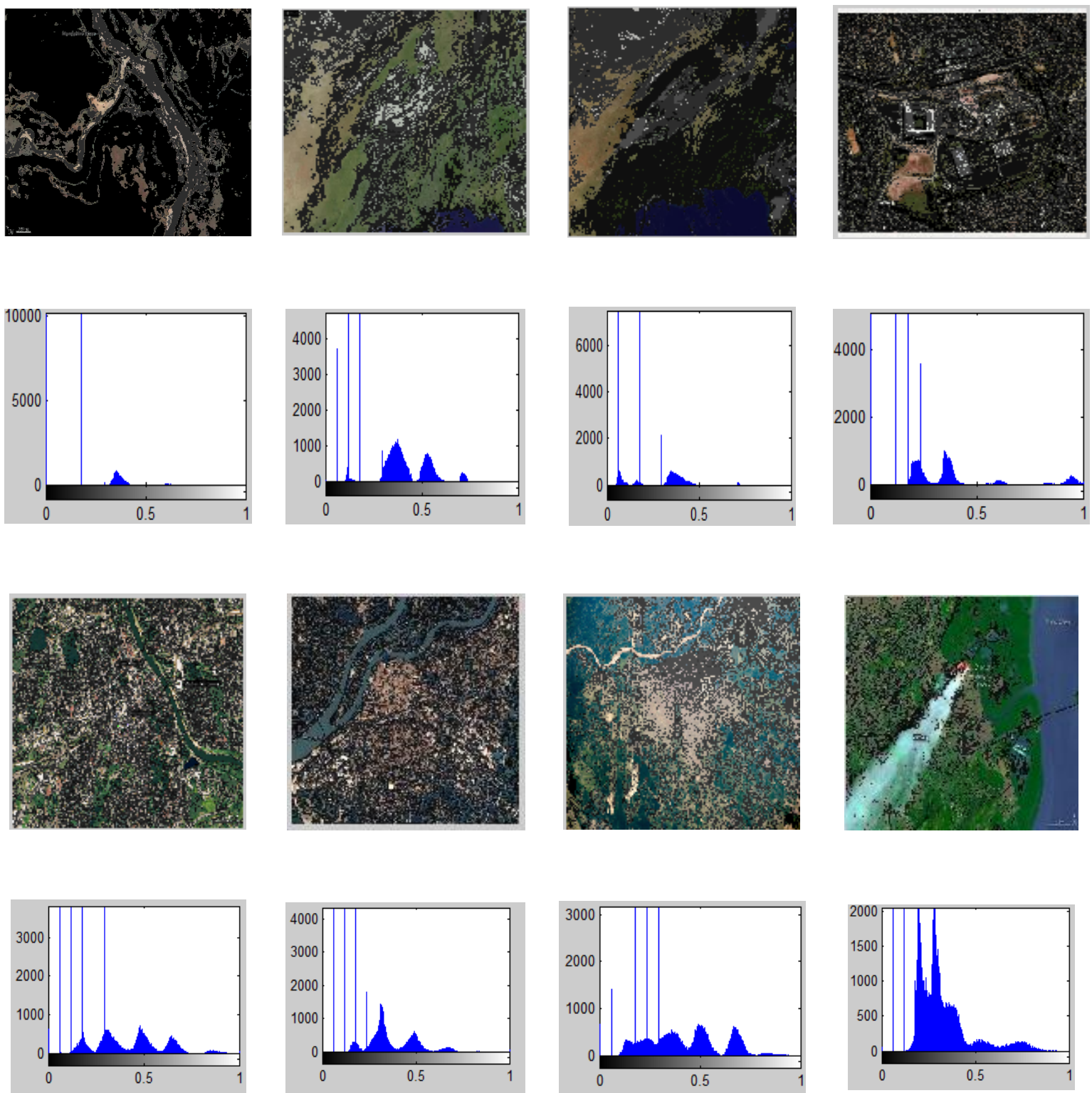

Figure 4. The 6 Levels Thresholding Based Segmented Images With Their Histograms 


\subsection{Threshold Levels 8}
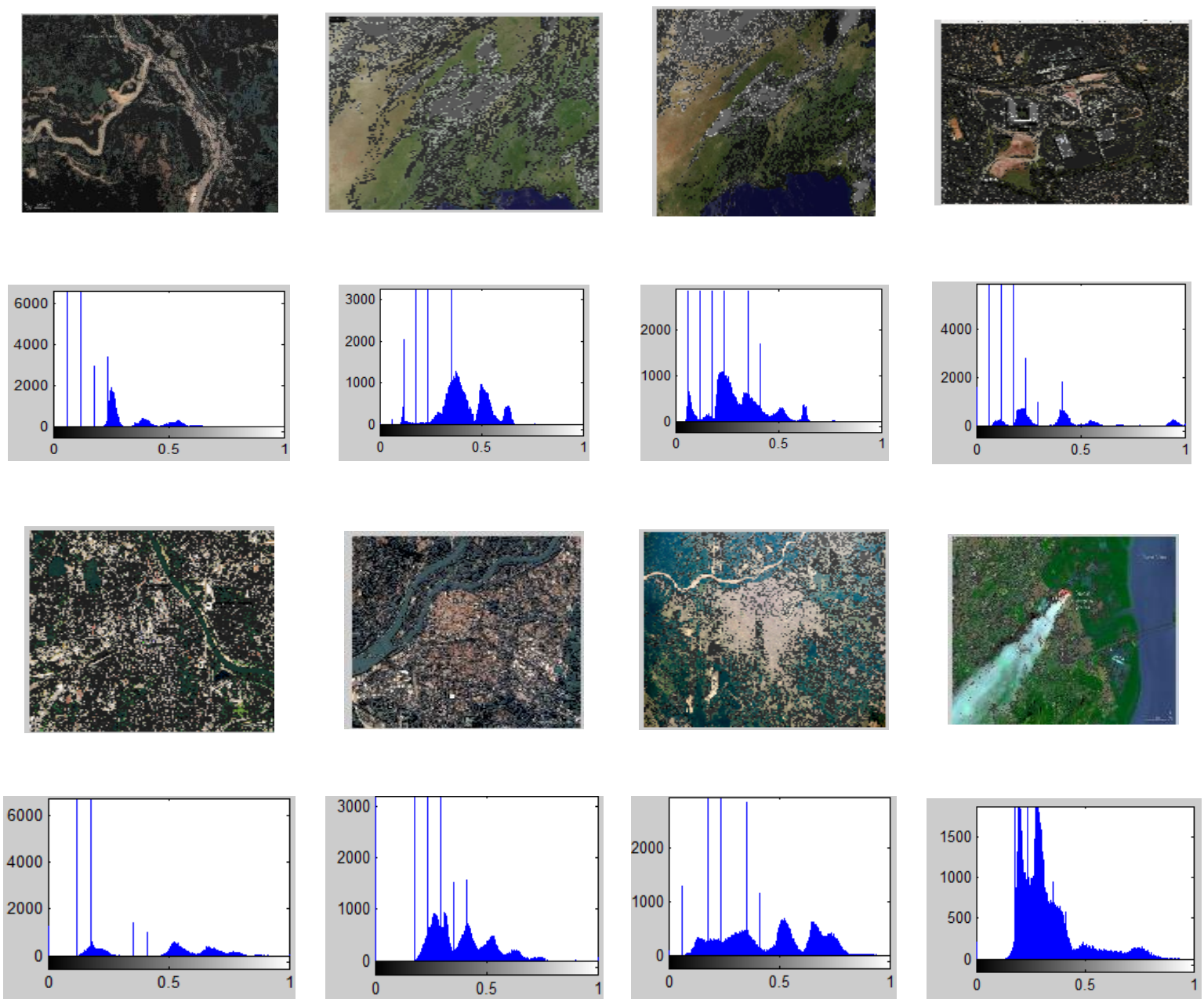

Figure 5. The 8 levels thresholding based segmented images with their histograms 


\subsection{Threshold Levels 12}
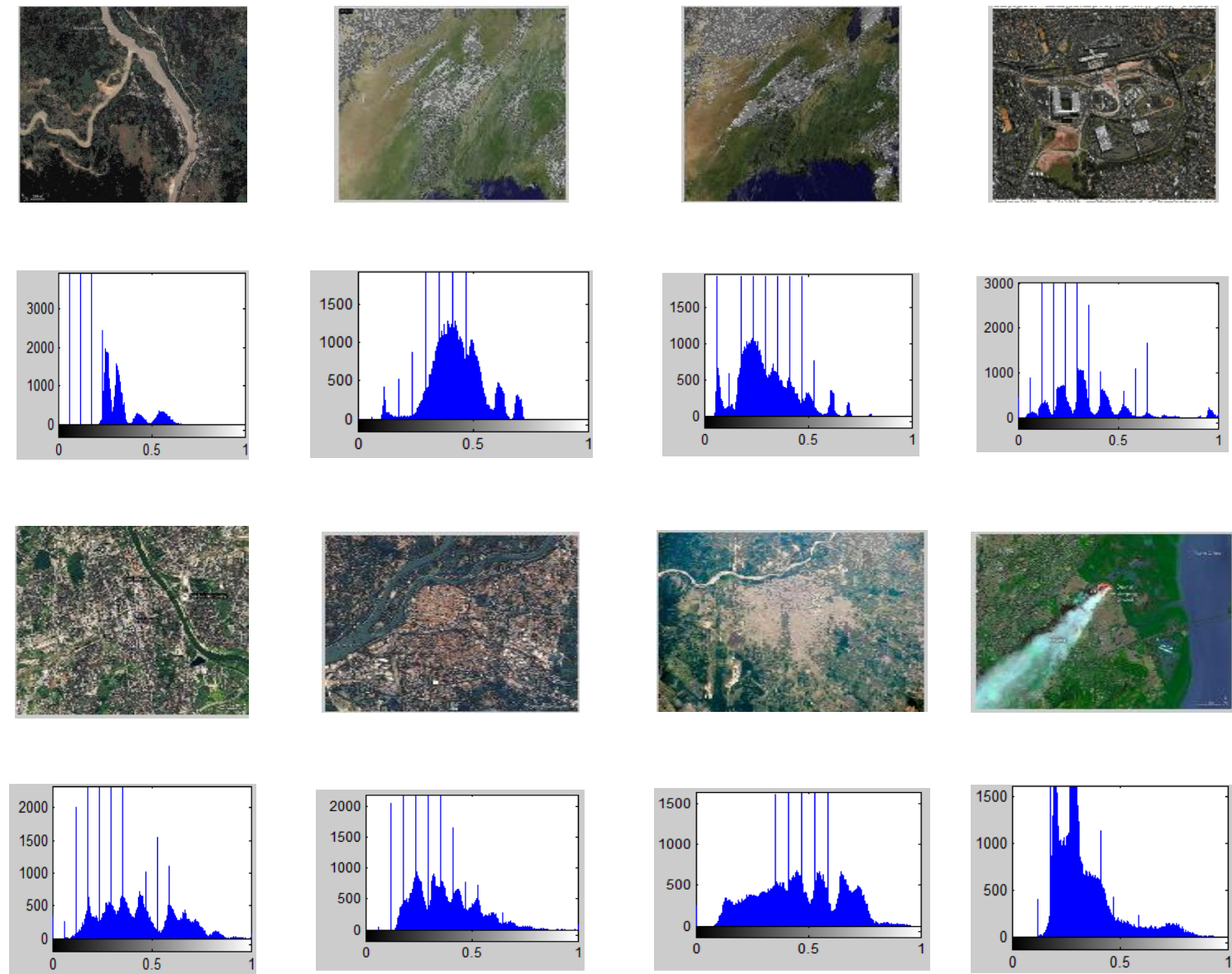

Figure 6. The 12 levels thresholding based segmented images with their histograms

\subsection{Quality Measurement Results}

The above Table contains matrices results of proposed algorithm and the algorithm has given the better results than the result of paper [1].

Table 2. Matrices of Proposed Algorithm

\begin{tabular}{|c|c|c|c|c|c|}
\hline Image No & Levels & MSE & PNSR & SSIM & $\mathrm{NCC}$ \\
\hline \multirow[t]{4}{*}{1} & 5 & 3.6 & 42.61 & 0.99 & 1.00 \\
\hline & 6 & 2.56 & 44.08 & 0.99 & 1.00 \\
\hline & 8 & 1.00 & 48.16 & 1.00 & 1.00 \\
\hline & 12 & 0.38 & 52.36 & 1.00 & 1.00 \\
\hline \multirow[t]{4}{*}{2} & 5 & 3.7 & 42.62 & 0.98 & 1.00 \\
\hline & 6 & 2.54 & 44.08 & 0.98 & 1.00 \\
\hline & 8 & 0.95 & 48.16 & 1.00 & 1.00 \\
\hline & 12 & 0.36 & 52.39 & 1.00 & 1.00 \\
\hline \multirow[t]{4}{*}{3} & 5 & 3.72 & 43.61 & 0.99 & 1.00 \\
\hline & 6 & 2.51 & 44.11 & 0.99 & 1.00 \\
\hline & 8 & 1.05 & 48.68 & 1.00 & 1.00 \\
\hline & 12 & 0.32 & 5365 & 1.00 & 1.00 \\
\hline \multirow[t]{4}{*}{4} & 5 & 2.16 & 44.81 & 1.00 & 1.00 \\
\hline & 6 & 2.23 & 44.68 & 1.00 & 1.00 \\
\hline & 8 & 1.23 & 47.25 & 1.00 & 1.00 \\
\hline & 12 & 0.25 & 54.22 & 1.00 & 1.00 \\
\hline \multirow[t]{4}{*}{5} & 5 & 3.74 & 42.44 & 0.99 & 1.00 \\
\hline & 6 & 2.84 & 43.63 & 0.99 & 1.00 \\
\hline & 8 & 2.07 & 45.00 & 0.99 & 1.00 \\
\hline & 12 & 0.32 & 53.16 & 1.00 & 1.00 \\
\hline \multirow[t]{4}{*}{6} & 5 & 3.82 & 42.34 & 0.99 & 1.00 \\
\hline & 6 & 2.23 & 44.69 & 1.00 & 1.00 \\
\hline & 8 & 0.75 & 49.42 & 1.00 & 1.00 \\
\hline & 12 & 0.17 & 56.87 & 1.00 & 1.00 \\
\hline \multirow[t]{4}{*}{7} & & 3.92 & 42.24 & 0.99 & 1.00 \\
\hline & 6 & 2.32 & 44.52 & 1.00 & 1.00 \\
\hline & 8 & 1.18 & 45.44 & 1.00 & 1.00 \\
\hline & 12 & 0.08 & 50.36 & 1.00 & 1.00 \\
\hline \multirow[t]{4}{*}{8} & 5 & 0.62 & 50.23 & 1.00 & 1.00 \\
\hline & 6 & 0.41 & 50.34 & 1.00 & 1.00 \\
\hline & 8 & 0.13 & 57.01 & 1.00 & 1.00 \\
\hline & 12 & 0.01 & 66.99 & 1.00 & 1.00 \\
\hline
\end{tabular}

Improved Color Satellite Image Segmentation Using Tsallis... (Jagan Kumar) 


\section{CONCLUSION}

In this proposed method the color satellite image segmentation has achieved the good results by the help of cuckoo search and that have supported by Tsallis entropy and Granular computing and because of this new algorithm the result have got the very optimized threshold values because of this optimized threshold values the execution time and computational cost have reduced to very lower and the segmentation of color satellite image have done effectively when compare to the previous algorithm[1,2].

\section{REFERENCE}

[1] P.D.Sathya And R.Kayalvizhi., "Optimum Multilevel Image Thresholding Based on Tsallis Entropy Method With Bacterial Foraging Algorithm".

[2] Hassan Id Ben Idder And Nabil Laachfoubi.,"Unsupervised Multilevel Thresholding Method for Weather Satellite Cloud Segmentation".

[3] Ashish Kumar Bhandari, Vineet Kumar Singh, Anil Kumar, Girish Kumar Singh, "Cuckoo Search Algorithm and Wind Driven Optimization Based Study of Satellite Image Segmentation For Multilevel Thresholding Using Kapur's Entropy".

[4] Hongbing Liu, Lei Li And Chang An Wu.,"Color Image Segmentation Algorithms Based on Granular Computing Clustering".

[5] Agrawal.S, Panda.R, Bhuyan.S \& Panigrahi.B.K.,'Tsallis Entropy Based Optimal Multilevel Thresholding Using Cuckoo Search Algorithm, Swarm and Evolutionary Computation", 2013.

[6] Bhandari.A.K, Kumar.A, Singh.G.K, "Tsallis Entropy Based Multilevel Thresholding For Colored Satellite Image Segmentation Using Evolutionary Algorithms", 2015.

[7] Ali.M., Siarry, P., \& Pant, M., "An Efficient Differential Evolution Based Algorithm for Solving Multi Objective Optimization Problems", 2012.

[8] Bhandari.A.K, Kumar,A., Singh,G.K., \& Soni,V.,"Performance Study of Evolutionary Algorithm for Different Wavelet Filters for Satellite Image Denoising Using Sub Band Adaptive Threshold", 2015.

[9] Bhandari,A.K, Soni,V, Kumar,A,\& Singh,G.K.,"Cuckoo Search Algorithm Based Satellite Image Contrast and Brightness Enhancement Using DWT-SVD”, 2014.

[10] Manikandan,S.,Ramar,K.,Willjuice,I.M.,\&Srinivasagan,K.G., "Multilevel Thresholding for Segmentation of Medical Brain Images Using Real Coded Genetic Algorithm”, 2014.

[11] Oliva,D., Cuevas,E., Pajares,G., Zaldivar,D., \& Perez-Cisneros,M.,’Multilevel Thresholding Segmentation Based on Harmony Search Optimization", 2013.

[12] Patra,S., Gautam,R., \& Singla,A.,"A Novel Context Sensitive Multilevel Thresholding for Image Segmentation”, 2014.

[13] Hammouche,K., Diaf,M., \& Siarry,P.,"A Multilevel Automatic Thresholding Method Based on A Genetic Algorithm for Fast Image Segmentation”,2008.

[14] Hamdaoui,F., Sakly,A., And Mtibaa,A.,"An Efficient Multilevel Thresholding Method for Image Segmentation Based on The Hybridization Of Modified PSO and Otsu's Method", 2015. 